\title{
Las comunicaciones electrónicas universitarias: el caso de la red académica de LUZ
}

\author{
Salom Arteaga, Rosa María*
}

\section{Resumen}

La comunicación como factor fundamental en el logro de los objetivos institucionales es analizada bajo la perspectiva de los retos que plantean las tecnologías de la comunicación electrónica. Se estudian con particular atención las características de uso de la Red Académica de la Universidad del Zulia y se define el perfil de su usuario por medio de un cuestionario enviado por correo electrónico. Los resultados obtenidos aquí son similares a los registrados en la Universidad de los Andes durante 1999, siendo el perfil del usuario el que demanda servicio, fundamentalmente, como un miembro del personal docente de sexo masculino, entre 36 y 50 años, que hace uso predominante del correo electrónico y de la búsqueda en Internet.

Palabras clave: Universidad, red académica, comunicación, usuario de nuevas tecnologías.

Recibido: 00-06-05. Aceptado: 00-10-13

Licenciada en Comunicación Social. Master in Radio \& Television. Master in European Business Communication. Profesora de la Universidad del Zulia. Coordinadora del Área de Nuevas Tecnologias del Centro de Investigaciones de la Comunicación y la Información de LUZ.

E-mail: rsalom@luz.ve.

Este estudio fue realizado gracias a los fondos suministrados por el Consejo de Desarrollo Científico y Humanístico de la Universidad del Zulia. La investigadora agradece la colaboración prestada por el Prof. Gustavo Vílchez, Coordinador de la Red Académica de LUZ. 


\title{
University Electronic Communications: The Academic Network in LUZ
}

\begin{abstract}
Communications, as a fundamental factor in achieving institutional objectives, is analyzed from the perspective of the challenges proposed by electronic communication technologies. Particular attention is paid to the use of the Academic Network in the University of Zulia, and a user profile is defined by means of a questionaire sent by electronic mail. The results obtained in this study are similar to those registered in the University of the Andes in 1999, where the user service profile was fundamentally, members of the professorate of masculine sex, between the ages of 36 and 50, and who principally utilize electronic mail and internet search.
\end{abstract}

Key words: University, academic network, communication, new technologies user.

\section{Introducción}

Debido a los crecientes costos en la educación superior, ésta se plantea como uno de sus dilemas la disminución de sus costos mientras mantiene la calidad. Pero para conquistar este reto, debe transformar la manera en que se desarrolla el proceso de aprendizaje.

Numerosos autores van más allá y señalan, como Hairston, que esta transformación será guiada por la necesidad de incrementar tanto el valor como la productividad a todos los niveles y en todos los miembros de la comunidad universitaria. $Y$ esto, añade, "requerirá pensamiento innovativo acerca de cómo pueden los estudiantes aprender mejor, cómo pueden los docentes guiarlos mejor hacia el aprendizaje y como los administradores pueden facilitar el ambiente más beneficioso" (Hairston, 1996:36). El autor seña- la que estos asuntos se trasladarán de la periferia del asunto a ser el tema central en la transformación exitosa del campus universitario.

La comunicación interpersonal juega un rol especialmente importante en las organizaciones, tanto como método de compartir y mantener culturas organizacionales (Schein, 1990), organizando y clarificando las tareas de grupo (Sundstrom, DeMeuse y Futrell, 1990) como reduciendo el estrés del trabajo (Bradley, 1989; Sauter, Murphy y Hurrell, 1990). La ausencia de un adecuado soporte social y comunicacional es particularmente visto como una fuente primaria de estrés en el trabajo (ver Sauter et al, 1990), y su mejora para el trabajo está relacionada con la resistencia al estrés, las enfermedades o el dolor (ver por ejemplo, Feuerstein, Sult y Houle, 1985; Quellete Kobasa y Puccetti, 1983; Caldwell y Taha, 1993). 


\section{La Comunicación y las Universidades}

Raisa Urribarri de Minardi y Orlando Villalobos, estudiaron en sus respectivas tesis de maestría, el problema de las comunicaciones en el ámbito universitario venezolano. Mientras Urribarrí de Minardi (1999) analizó el uso de las nuevas tecnologías de la comunicación por los docentes de la Universidad de los Andes, Villalobos (1998:15), quien concentró sus esfuerzos en la comunicación de la Universidad del Zulia, anotaba que:

El déficit comunicacional, resultante de la desinformación que predomina en LUZ, im. pide que la filosofía de gestión que orienta el quehacer de la universidad se concrete, reduce las posibilidades de éxito del gobierno y del gobiemo universitario, al quedar los organismos de dirección sin la suticiente vinculación con la comunidad.

Muy acertadamente, Villalobos (1998:46) apuntaba que cuando la comunicación, institucional y científica, no cumple su objetivo dentro de la institución, se dificulta el consenso entre los distintos actores y no se da la suficiente o necesaria integración, adaptación, logro de objetivos, mantenimiento de pautas y control de tensiones. Esto ocurre por la inexistencia de intercambios de información.

En su tesis de grado, Villalobos (1998:15) señala tres factores que se suman para obstaculizar una eficiente comunicación universitaria: Ausencia de una concepción de la comunicación válida para toda la universidad (es lo que frecuentemente se denominan políticas 0 estrategias). Poca claridad de objetivos de la gerencia en materia de comunicación. Poca integración de los recursos humanos.

El trabajo de Villalobos (1998:117) incluye una encuesta realizada entre los miembros de la comunidad de LUZ. Más de la mitad de los entrevistados estima que esa casa de estudios no posee una política orgánica definida y el autor concluye que "... si bien LUZ cuenta con diversos medios, recursos humanos y una determinada infraestructura tecnológica, que busca resolver los requerimientos comunicacionales e informativos de su comunidad, sin embargo, de acuerdo con este estudio, en ésta prevalece la desinformación".

La reciente celebración del primer centenario del periódico de la Universidad del Zulia fue marco oportuno para la difusión de las diferentes perspectivas con las que esta universidad enfrenta los retos que plantea su problemática comunicacional. No fue una mera coincidencia que casi todos los lideres consultados por el periódico de LUZ coincidieran en la necesidad de estimular y garantizar un democrático diálogo intra-universitario, fortalecer sus comunicaciones internas $y$ externas y hacer más eficiente el aparato tecnológico que permita estos logros (La Universidad del Zulia, 1998).

Sin embargo, persisten la ineficiencia comunicativa, la duplicación de esfuerzos y la sensación de que "cada quien va por su lado" en materia de comunicación. Por ejemplo, existe un notorio retraso en la aplicación de nuevas tecnologias a la producción y distribución de información en pantalla en la Universidad del Zulia, especialmente en el servicio bibliotecario. Este retraso contri- 
buye a la ineficiencia de la institución en términos administrativos y entorpece el desarrollo de sus actividades primarias: la docencia y la investigación, un problema presente en todas las universidades venezolanas con diversos niveles de gravedad en cada caso.

\subsection{Las interrogantes sobre la comunicación electrónica}

Por definición, cualquier tecnología o medio de comunicaciones actúa para producir contacto entre personas, a través del compartir ideas o símbolos. Este contacto puede ser inmediato, recíproco y evidente como los contactos cara-a-cara, o puede ser muy distante en tiempo y lugar, y unidireccional como por ejemplo, un artículo en el periódico o un programa grabado de televisión. Las modernas tecnologías de comunicación, especialmente las electrónicas basadas en el computador, buscan incrementar el contacto y, por lo tanto, disminuir la "distancia" entre personas -tanto física como temporal o social- a través del medio de comunicación (Caldwell y Taha, 1993).

El vertiginoso crecimiento de las comunicaciones electrónicas y particularmente de Internet ha provocado un aumento en los estudios sobre el impacto de las comunicaciones electrónicas y particularmente Internet en los ambientes Universitarios: en enero de 1999 existían en el mundo 43,230,000 hosts (Internet Software Consortium: 1999). Al año siguiente, ese número se había elevado a $72,398,000$ (Internet Software Consortium, 2000).
Las barreras sociales, organizacionales y tecnológicas para implementar y evaluar sistemas de comunicación a través de computadores (CMCS en inglés) $u$ otros medios electrónicos, generan significativos problemas técnicos y sociales a los diseñadores, administradores y gerentes del sistema, asi como a los investigadores de la conducta (Bradley, 1989; Eason, 1989; Olson, 1989). Algunos investigadores sugieren que alrededor de $20 \%$ de los sistemas implementados son exitosos (Eason, 1989).

Entre los factores principales que contribuyen a los fracasos y reducen la productividad encontramos: a) pobreza de la integración del CMCS en las actividades de la organización o resistencia cultural a la adopción del CMCS (Caldwell, 1992; Eason, 1989; Lowry, 1992) y b) inadecuación de la planificación o instalación del sistema (Maryniak y Caldwell, 1992, Caldwell y Taha), 1993).

Los medios de comunicación proveen montos variados de contenido de información debido a las restricciones en el modo o tipos de información que pueden ser compartidos a través del medio (Daft y Lengel, 1984; Rice, 1984; Short, Williams y Christie, 1976). Por ejemplo, los mensajes escritos tienen limitaciones para comunicar muchas claves no verbales como la entonación, la postura del cuerpo o las expresiones faciales. Numerosos indices han sido propuestos para cuantificar la capacidad de varios medios de comunicación para servir de soporte al proceso de comunicación. Short et al (1976) plantean la discusión en términos de "presencia social", lo cual está relacionado con características per- 
ceptuales y afectivas como calidez y soporte para la interacción personal y sensitiva (ver también Fulk, Steinfield, Schmitz y Power, 1987).

Una visión alternativa caracteriza los medios en términos de "riqueza informativa" (Daft y Lengel, 1984 y 1986; Trevino, Lengel y Daft, 1987). Rice (1984) usa el término "ancho de banda" para describir conceptos similares. La riqueza es definida primariamente a través de características objetivas y técnicas del medio, incluyendo velocidad de la comunicación de retorno (feedback) y capacidad de transmisión de información de los canales disponibles del medio (Caldwell y Taha, 1993).

Existen numerosos estudios que enfocan el dónde y cómo es utilizado el CMCS en las organizaciones y la efectividad de la tecnologia del CMCS (ver, por ejemplo, Balasubramanian, 1987; Hiltz y Johnson, 1990; Papa y Papa, 1990; Rice, 1984; Rice y Williams, 1984; Rice et al, 1990). En el contexto de la reducción del aislamiento social, un CMCS es efectivo en la medida en que permita a las personas lograr niveles deseados de contactos relativos a sus necesidades de información e interacción social o profesional (Caldwell y Taha, 1993).

En algunos casos, la dificultad en el acceso o uso del sistema puede convertirse en una barrera para el uso efectivo del CMCS (Eason, 1988; Rice y Shook, 1988); el soporte organizacional y social hacia el CMCS también influye significativamente en su uso (Blomberg, 1987; Rice y otros, 1990; Taylor, 1987). Algunas investigaciones han demostrado que los procesos de desarrollo de normas de grupo y la toma de decisiones grupales son afectados por las claves transmitidas a través del medio (Archer, 1990; Spears, Lea y Lee, 1990).

Está claro que la comunicación por medios electrónicos como método exclusivo de interacción no será aceptado por muchos trabajadores, tal como ha sido visto por las dificultades para implementar sistemas completos de telecomunicaciones (Mokhtarian, 1991). Una razón importante para este rechazo generalizado es el aislamiento social que resulta de las restricciones en los contactos cara a cara, informales y no planificados, con los compañeros de trabajo (Byte, Roundtable, 1991; Sproull y Kiesler, 1991).

Para maximizar la productividad, la tecnología del CMCS debe adecuarse apropiadamente a las necesidades comunicacionales de una organización y permitir los tipos y modos deseados de comunicación con un mínimo de restricciones percibidas y costos organizacionales y económicos (Eason, 1988).

\subsection{Las Comunicaciones Electrónicas en el ámbito universitario}

El trabajo de Silvio (1998) muestran una clara tendencia hacia un uso masivo de las nuevas tecnologias de la información y la comunicación por las universidades $(60 \%$ a nivel mundial y más de la mitad en las Latinoamericanas y del Caribe). En Venezuela, a finales del siglo pasado casi todas las universidades públicas y más de la mitad de las privadas, poseian una página web en Internet, cuyo propósito principal era proveer información sobre las casas de estudios superiores (Salom, 1999). Sin embargo, se requiere estudiar más 
profundamente, las caracteristicas internas de los usos de las tecnologías de la información y la comunicación en cada universidad venezolana.

El estudio de Raisa Uribarrí de Minardi (1999) sobre el uso que hacen los docentes de los sistemas informáticos de la Universidad de los Andes, es un paso importante en la búsqueda de un mecanismo que permita incorporar de manera eficiente las comunicaciones en la comunicación universitaria. El trabajo de Urribarri de Minardi indica claramente que un porcentaje reducido del personal docente hace uso intensivo de las nuevas herramientas de comunicación, que el uso se concentra en el correo electrónico y búsqueda de Internet y que el valor agregado, entendido como la cantidad de información que los académicos colocan en la web, es mínimo.

Estos hallazgos de Urribarri son similares a los encontrados en 1997 por $T$. Matthew Ciolek (1998). El autor realizó una encuesta sobre el Uso que hacen los Académicos de la Web. La encuesta fue enviada a 1767 suscriptores de siete listas de correo electrónico de profesores de Inglés especializados en Estudios Asiáticos y del Pacífico. Los datos indican que los individuos dedicados predominantemente a actividades de investigación y de educación universitaria tenian en 1997 un promedio de 4 años de experiencia con Internet y trabajan más de 4 horas diarias en la Net.

Los resultados de Matthew indican que los tres usos más populares de internet se encuentran en el envío y recepción de correo electrónico y la lectura de noticias y que las actividades profesionales más frecuentes, relacionadas con Inter- net, son aquellas que envuelven el desarrollo de páginas personales, la investigación en Internet y el mantenimiento de las listas de correo electrónico.

\section{Los Usuarios de la Red Académica de LUZ}

Durante el mes de marzo de 1999, los usuarios de la Red Académica de la Universidad del Zulia recibieron a través de correo electrónico una encuesta que permitiría conocer el perfil del usuario de este servicio. De un total de 1500 usuarios inscritos en aquel momento en la red (menos del $25 \%$ del personal docente de esa casa de estudios), sólo 46 respuestas fueron recibidas durante los siguientes 15 días después de enviada la encuesta. Esta cantidad de respuesta, equivalía al $3.06 \%$ de los usuarios inscritos. Sin embargo, es importante anotar que la investigadora recibió correos adicionales de cerca de 15 miembros de la red que acusaron problemas para el envío de las respuestas, problemas de carácter técni$\mathrm{co}$, atribuidos por los usuarios al servidor. No resulta, por tanto, aventurado suponer que el número de respuestas pudo ser muy superior si los problemas técnicos no se hubieran presentado.

Exactamente un año más tarde se repitió el envio del cuestionario (en esta oportunidad más reducido) y se siguió la metodología aplicada por Urribarrí de Minardi (1999) de enviar el cuestionario en tres oportunidades en un lapso de tres semanas. Se recibieron 148 respuestas las cuales fueron contrastadas con las del año 1999 con el propósito de verificar la evolución de la Red Académica de LUZ. 
Las respuestas obtenidas en ambos casos, nos permiten inferir algunas características relativas al usuario de la Red Académica de LUZ. Lo primero es que este usuario se identifica primariamente como docente. Tanto en la medición del año 1999 como en la del año 2000 , más del $80 \%$ de las respuestas pertenecen al personal docente (ver gráfico 1).

Los resultados del siguiente año (2000) muestran que a pesar de que el porcentaje de docentes $(83 \%)$ se mantiene igual, el de empleados se incrementa en más del doble y el de estudiantes se reduce a menos de la mitad con relación al año anterior. El incremento en el número de empleados podria atribuirse a la incorporación de algunas bibliotecas y dependencias administrativas, mayormente del Vice-rectorado Académico, a la Red Académica.

La encuesta realizada durante 1999 (ver gráfico 2) mostraba que la facultad de Humanidades poseía mayor número de usuarios que otras facultades tradicionalmente asociadas con los re- cursos telemáticos como Ingeniería y Ciencias. Esto podría ser motivado a la inauguración ese año del Centro Tales y del servidor de esa facultad.

Un fenómeno similar aparece durante el año 2000 (ver gráfico 2). Mientras Ingeniería aparece en primer lugar en número de usuarios, Humanidades retrocede al segundo, Ciencias asciende al tercero y surge la Facultad de Arquitectura como la de mayor crecimiento en número de usuarios durante el último período. Nuevamente coincide este crecimiento con la puesta en servicio recientemente, del servidor de esa facultad.

Los resultados reflejan una concentración del $85 \%$ de los usuarios en cinco dependencias: Ingeniería, Humanidades, Ciencias, Arquitectura y Rectorado. El restante $15 \%$ se distribuye entre Medicina, Cs. Jurídicas, Veterinaria, Agronomía y Odontología (no se recibieron respuestas de la facultad de $\mathrm{Cs}$. Económicas y Sociales).

Con el propósito de verificar el crecimiento o no de la red académica, se in-

\section{Gráfico 1}

Universidad del Zulia

Usuarios de la Red Académica

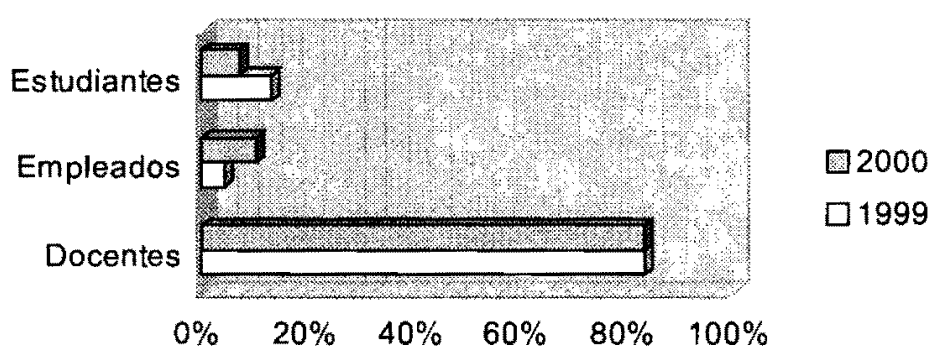

Fuente: Encuesta propia. 


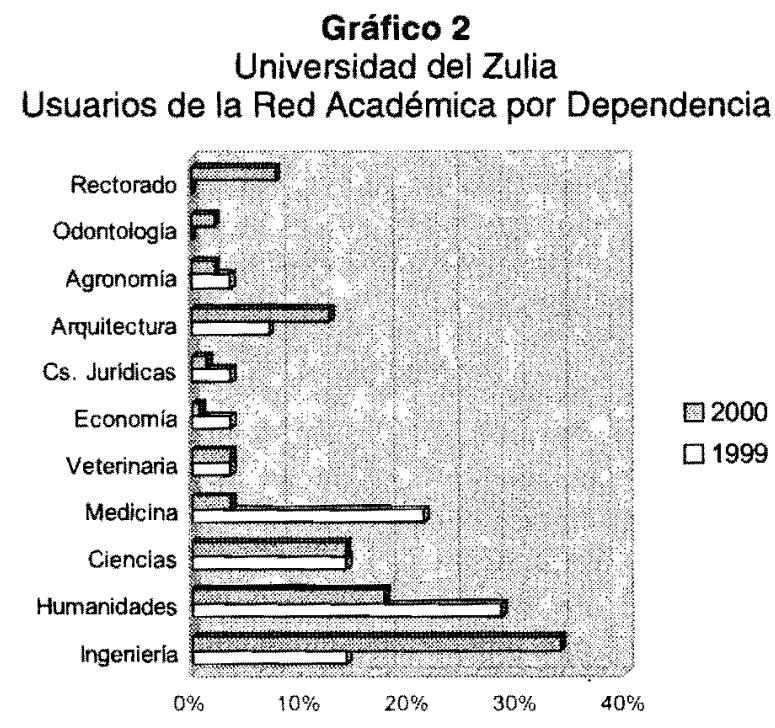

Fuente: Encuesta propia.

cluyó en ambos cuestionarios una pregunta relativa a la antigüedad en la red. Los resultados de 1999 revelan que más de las tres cuartas partes pertenecen a la red desde hace más de 1 año. Para el 2000, los usuarios declaran, en su mayoría, tener más de dos años en la RA (ver gráficos 3 y 4). Estos datos nos permiten inferir un crecimiento menor al $10 \%$ en el número de usuarios del servicio durante los últimos tres años.

Una de las incógnitas a resolver era la frecuencia de uso. Los resultados de los años 1999 y 2000 (gráfico 5) muestran como los usuarios hacen uso diario de los diversos servicios, lo cual indicaría el carácter fundamental que hacen de la telemática.

Esto ratificaria su condición de usuarios asiduos, aunque se observaron diferencias: los resultados del año 1999 revelan que mientras la gran mayoría de los docentes utilizaban el servicio todos los días, en el caso de los estudiantes el uso se reducía a 2 ó 3 veces por semana. Para el año 2000, estas frecuencias cambian, en el sentido de que todos los usuarios (docentes, empleados y estudiantes) hacen un uso mayoritariamente diario del servicio. Este cambio es particularmente dramático en el caso de los estudiantes, los cuales declaran en su casi totalidad $(89 \%)$ utilizar el servicio todos los días, superando en ello incluso a los docentes.

Otro de los patrones de uso estudiados fue el tiempo de conexión. Mientras en 1999 la gran mayoría de los docentes se conectan por periodos superiores a una hora, la mitad de los estudiantes que respondieron al cuestionario to hacian durante lapsos que no superaban la media hora. Estos hábitos se han modificado ligeramente durante el siguiente lapso (ver gráfico 6). 


\section{Gráfico 3}

Red Académica de La Universidad del Zulia Antigüedad como usuarios (1999)

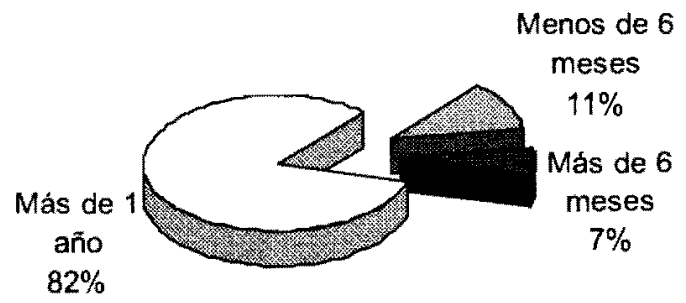

Fuente: Encuesta propla.

\section{Gráfico 4}

Red Académica de La Universidad del Zulia Antigüedad como usuarios de RA (2000)

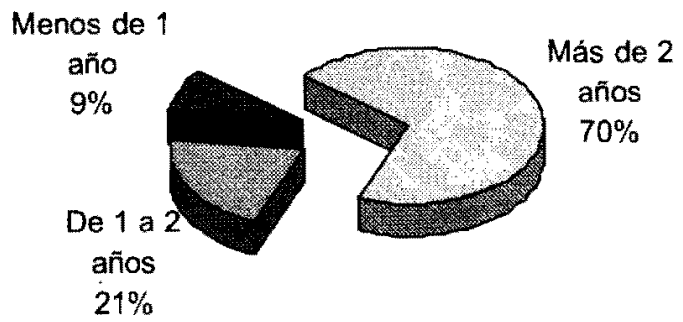

Fuente: Encuesta propia.

\section{Gráfico 5}

Universidad del Zulia

Frecuencia de conexión a la Red Académica

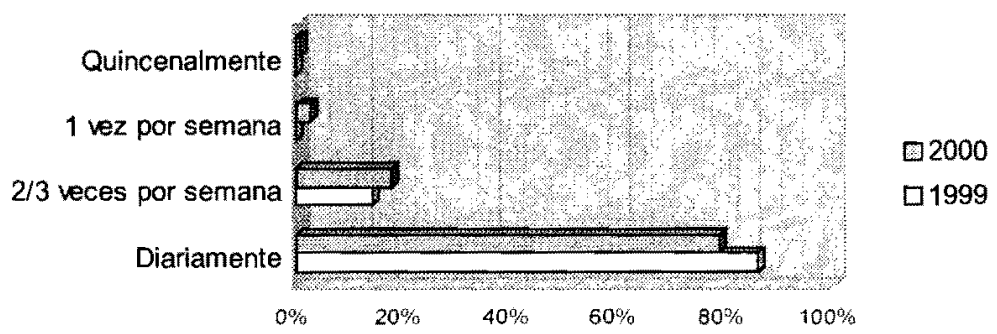

Fuente: Encuesta propia. 
Comunicaciones electrónicas universitarias: red académica de LUZ Salom Arteaga, Rosa Maria

Indagamos si estos patrones de tiempo de conexión estaban relacionados con la pertenencia a una dependencia universitaria particular. Los resultados del año 1999 muestran que en general, los de Ciencias e Ingeniería son los que se conectaban menos tiempo $(75 \%$ indican 60 minutos o menos), y los que se mantenian mas tiempo en línea eran los de Humanidades. En general, la mayoria de los usuarios estaban conectados durante mas de 1 hora diaria (ver Figura 6). Este patrón se mantiene durante el año siguiente y observamos que el
$50 \%$ de los docentes se conectan durante más de 30 minutos, de ellos, más de un tercio lo hace durante más de una hora (ver gráfico 7 ).

Durante el año 2000 se mantiene la tendencia observada durante el año anterior: los usuarios de Humanidades son los que se mantienen conectados mayor número de minutos, seguidos de Medicina e Ingeniería.

Al tratar de definir el Perfil del Usuario de la Red Académica de LUZ se buscaba verificar si existian tendencias relacionadas con el horario de utilización de

\section{Gráfico 6}

Universidad del Zulia

Tiempo de conexión a la Red Académica

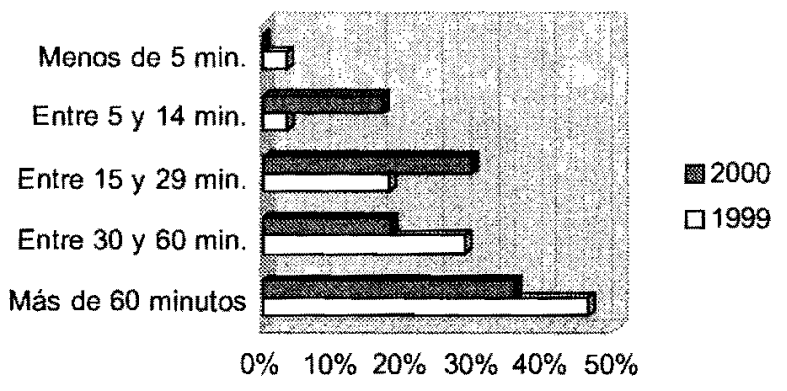

Fuente: Encuesta propia.

\section{Gráfico 7}

Red Académica de La Universidad del Zulia

Tiempo de conexión de docentes (2000)

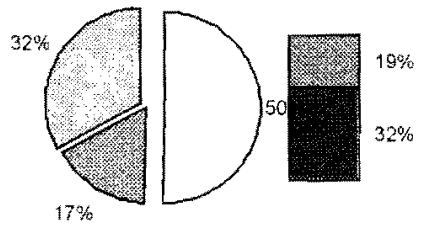

Fuente: Encuesta propia. 
los servicios. Los hallazgos del año 1999 (ver gráfico 8), aunque no son concluyentes, parecen indicar que la mayoría no tiene horario definido, sin embargo, al contrastar estos resultados con los del año siguiente, se observa una marcada concentración de los usuarios en el horario matutino (gráfico 8). Se observaron algunos casos consistentes en las facultades de Medicina y Ciencias que parecieran señalar cierta coincidencia con los horarios regulares de permanencia docente $(8$ $A M$ a $12 m$ y 2 a 6 PM).

Una de las hipótesis del estudio indicaba que el correo electrónico sería el servicio más utilizado al igual que la búsqueda en Internet. Los resultados confirmarian tal hipótesis (ver gráfico 9).

Durante el año 2000, se confirmó la tendencia de utilizar mayoritariamente el servicio de Correo Electrónico $92 \%$, búsqueda en Internet $(88 \%)$, conexión con otras universidades $(60 \%)$, lectura de noticias $(44 \%)$, FTP $(30 \%)$, realización de compras a través de Internet $(15 \%)$.

Otra de las categorías indagadas durante 1999 fue el lugar desde donde el usuario accesa el servicio. Mientras que todos los encuestados de Ingeniería, Ciencias Jurídicas, Agronomia y Arquitectura señalaron que se conectaban desde su oficina en LUZ, la mitad de los usuarios de Medicina lo hacen desde su hogar, asi como un tercio de los de Humanidades (ver gráfico 10). Esta variable no fue medida durante el año 2000 pues la administración de la RA desactivó la posibilidad de acceso de la mayoría de los servicios fuera del campus universitario y por ello no podemos suministrar datos comparativos, por lo que suponemos que la casi totalidad de las consultas se realizan desde el sitio de trabajo en LUZ.

Al solicitar a los usuarios que calificaran la calidad del servicio prestado por la Red Académica, la gran mayoría lo describió como eficiente durante el año 1999 (ver gráfico 11). Estos datos resultan paradójicos si tomamos en cuenta el frecuente nủmero de interrupciones en el servicio, las restricciones de acceso y la limitada capacidad de los servidores, por mencionar algunos problemas. Sin em-

\section{Grático 8}

Universidad del Zulia

Horario de utilización de la Red Académica

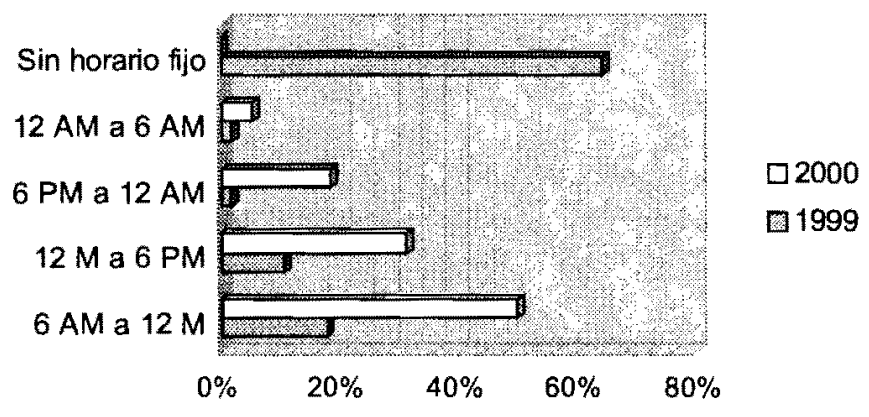

Fuente: Encuesta propia. 


\section{Gráfico 9}

Red Académica de La Universidad del Zulia

Servicios más utilizados

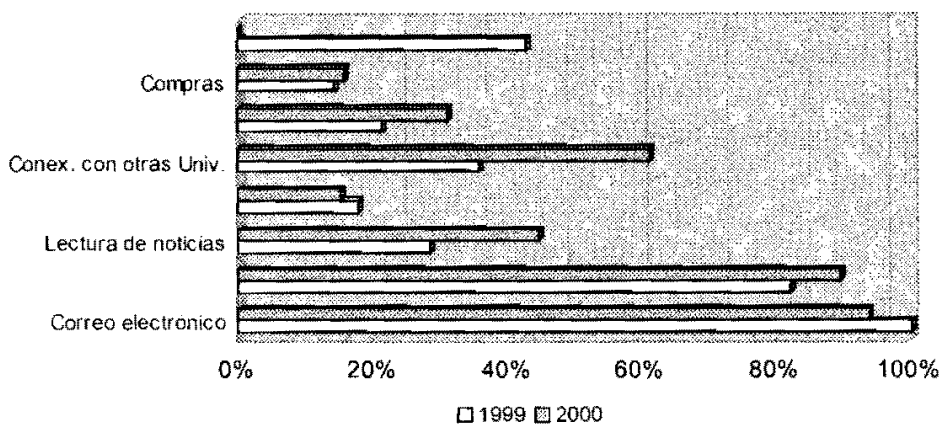

Fuente: Encuesta propia.
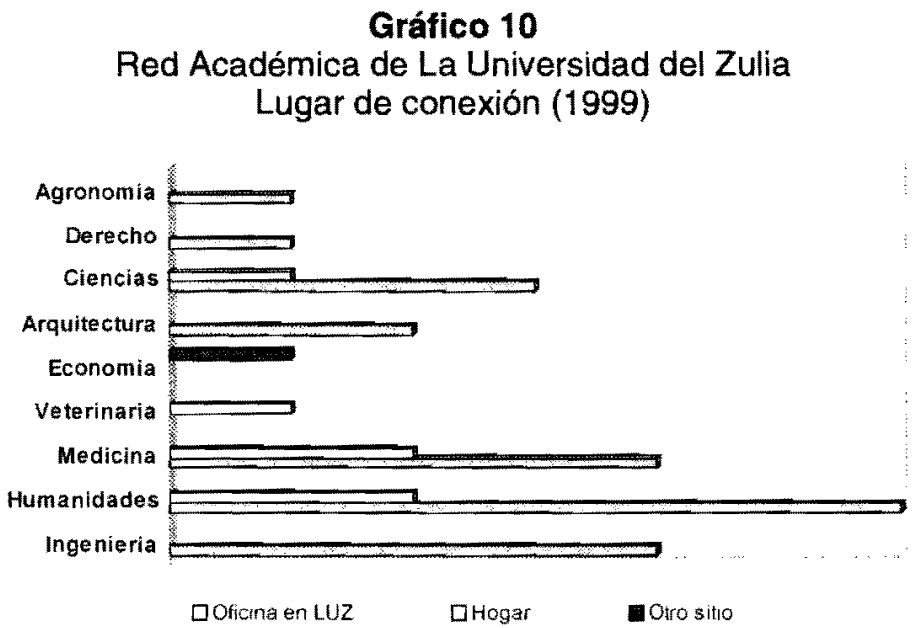

Fuente: Encuesta propia.

bargo, al comparar los resultados del año 1999 con los del siguiente año, se verifica un creciente descontento en relación a la satisfacción generada por el servicio, pues se incrementa dramáticamente el porcentaje de usuarios que califica el servicio como deficiente o muy deficiente y disminuye el número de respuestas de usuarios que califican el servicio como eficiente o muy eficiente (ver gráfico 11).

La indagación indica que hubo un incremento en el porcentaje de usuarios del sexo masculino que pasaron del $60 \%$ en 1999 al $70 \%$ al año siguiente (ver 


\section{Gráfico 11 \\ Universidad del Zulia \\ Calificación de la Red Académica por sus usuarios}

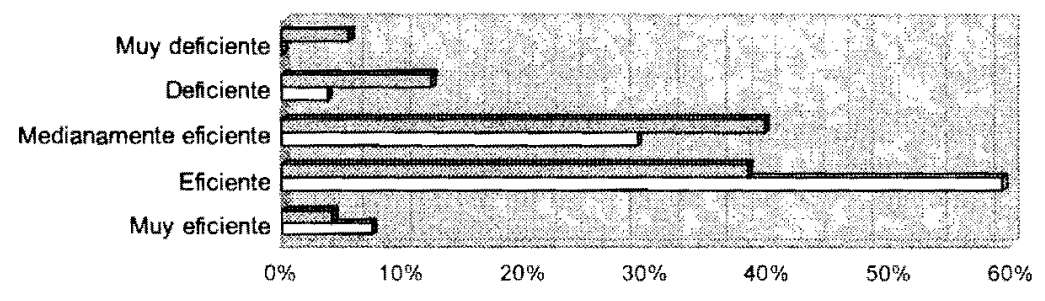

ㅁ1999 2000

Fuente: Encuesta propia.

gráfico 12). Paradójicamente, mientras el número de estudiantes del sexo femenino crece en la Universidad del Zulia, el porcentaje de usuarios de la Red Académica de ese sexo, descendió del $40 \%$ en 1999 , al $30 \%$ para el año siguiente.

Otra variable independiente estudiada fue la edad de los usuarios (ver gráfico 13). Para el año 1999 , el $67 \%$ de los usuarios tienen edades comprendidas entre los 36 y 50 años. Al año siguiente el porcentaje de usuarios de esa categoría de edad desciende hasta un $50 \%$. El porcentaje de usuarios de edades comprendidas entre 25 y 35 años se redujo a la mitad entre 1999 y el año siguiente. Resulta por demás curioso que la reducción más dramática se observó en las respuestas de los usuarios menores de 24 años: mientras en 1999 ellos constituian el $7 \%$ de las respuestas, para el año siguiente ese porcentaje se había reducido hasta un $1 \%$. Los mayores de 50 años no presentaron mayores fluctuaciones entre un año y otro ( $14 \%$ en 1999 y $13 \%$ en el 2000$)$.

\section{Conclusiones}

Mientras se observa una tendencia mundial hacia el uso generalizado de las herramientas de comunicación electrónicas, particularmente de Internet, en las labores docentes y de investigación, en la Universidad del Zulia estos recursos se mantienen restringidos a una elite de profesores que no sobrepasa el $25 \%$ de la academia. El servicio de la Red Académica está, por definición, orientado a auxiliar las actividades de investigación, con insignificante acceso al personal administrativo y cuerpo estudiantil.

El estudio realizado permite inferir que el usuario de la Red Académica de la Universidad del Zulia pertenece al personal docente $(83 \%)$, mayoritariamente del sexo masculino, con una edad comprendida entre 36 y 50 an̆os, adscrito a las facultades de Ingeniería o Humanidades, el cual hace uso diario del servicio y se mantiene conectado durante más de 60 minutos en cada ocasión, tiene más de dos 
Comunicaciones electrónicas universitarias: red académica de $L U Z$

Salom Arteaga, Rosa María

Gráfico 12

Red Académica de La Universidad del Zulia

Distribución de usuarios (sexo)

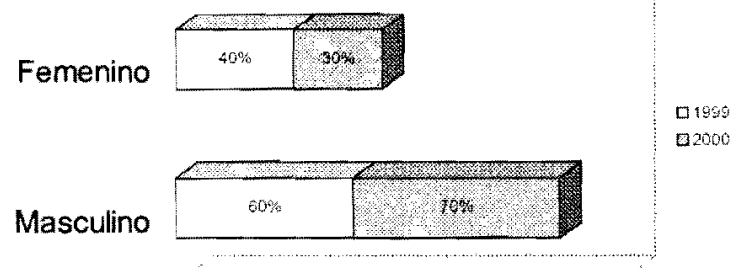

Fuente: Encuesta propia.

\section{Gráfico 13}

Universidad del Zulia

Edad de usuarios de la Red Académica

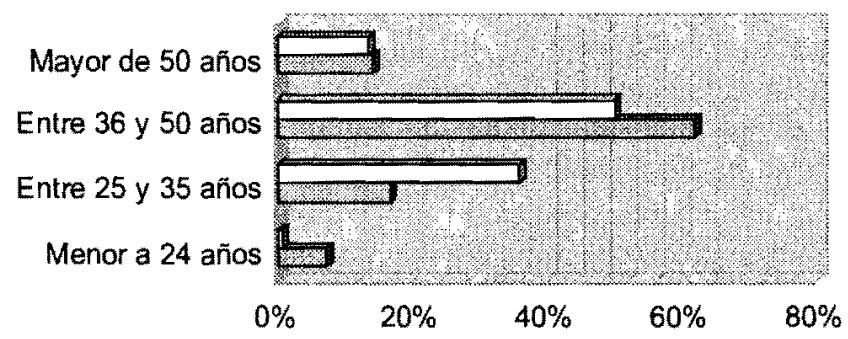

Fuente: Encuesta propia.

años de antigüedad como usuarios, se encuentra medianamente satisfecho con respecto al servicio prestado por la red y hace uso fundamental del correo electrónico y la búsqueda en Internet.

La incógnita se plantea en términos de los objetivos que persigue la RA y su rol dentro de la política de comunicaciones de LUZ. Precisamente dentro de los objetivos que se plantea la Coordinación de la RA, no se incluyen la promoción, gestión y desarrollo de los recursos telemáticos fuera del área de la investigación, pues tal como lo expresa claramente su reglamento, la Red Académica: "Dotará a los equipos de investigación de los equipos necesarios para su organización, financiamiento y desarro"lo" (Coordinación Red Académica, 2000).

Cabria suponer entonces que la Universidad del Zulia, deliberadamente ha excluido los recursos de la Red Académica del proceso de enseñanza aprendizaje. ¿Puede esto justificarse? ¿Es acaso saludable excluir los recursos telemáti- 
cos de la política de comunicaciones de una institución?

La nueva Ley de Telecomunicaciones y el reciente anuncio del Ejecutivo que declara el acceso a Internet como materia de interés nacional, especialmente para el sector educativo, obligará a las universidades nacionales, entre ellas a LUZ, cuyo servicio ha experimentado un crecimiento excesivamente modesto en los últimos tres años, a realizar una profunda revisión del rol que las nuevas tecnologias de la información y la comunicación juegan en el desarrollo de los fines y objetivos de la educación superior venezolana.

\section{Bibliografía citada}

Archer, N. P. (1990). "A Comparison of Computer Conferences with Face-to-face Meetings for Small Group Business Decisions." Behaviour and Information Technology, en CALDWELL, Barrett y TAHA, Lilas (1993).

Balasubramanian, J. (1987). "Influence of Status on Group Interaction Processes: Electronic Mail versus Face-to-Face Discussions." Proceedings of the Human Factors Society en CALDWELL, Barrett y TAHA, Lilas (1993).

Blomberg, J. L. (1987). Social Interaction and Office Communication: Effects on User's Evaluation of New Technologies en CALDWELL, Barrett $y$ TAHA, Lilas (1993).

Bradley, G. (1989). Computers and the Psychosocial Work Environment. en: CALDWELL, Barrett y TAHA, Lilas (1993).

Byte, Roundtable (1991). "Is It Time To Telecommute?" en CALDWELL, Barrett y TAHA, Lilas (1993).

Caldwell, B.S. (1992). The role of Socio-Technical Systems Engineering in Im- plementing Office Automation and Information Technologies en CALDWELL, Barrett y TAHA, Lilas (1993).

Caldwell, Barrett y Taha, Lilas (1993). "Starving at the Banquet: Social Isolation in Electronic Media". Interpersonal Computing and Technology: An Electronic Journal for the 21st Century, January, Vol. 1, \# 1. http: www.helsinki.fi/science/optek/1994/ N2/ caldwell.txt (23 de Marzo 1998).

Coordinación de la Red Académica (2000). Página Principal de la Universidad del Zulia en http://www.luz.ve (Junio 1 , 2000).

Daft, R. L., \& Lengel, R. H. (1984). Information Richness: A New Approach to Managerial Behavior and Organization Design en CALDWELL, Barrett y TAHA, Lilas (1993).

Daft, R. L., \& LengeL, R. H. (1986). "Organizational Information Requirements, Media Richness and Structural Design." en CALDWELL, Barrett y TAHA, Lilas (1993).

Eason, K., (1988). Information Technology and Organizational Change. en: CALDWELL, Barrett y TAHA, Lilas (1993).

Feuerstein, M., Sult, S., \& Houle, M. (1985). "Environmental Stressors and Chronic Low Back Pain: Life Events, Family and Work Environment." en: CALDWELL, Barrett y TAHA, Lilas (1993).

Fulk, J., Steinfield, C.W., Schmitz, J., Y Power, J. G. (1987), "A Social Information Processing Model of Media Use in Organizations." en CALDWELL, Barrett y TAHA, Lilas (1993).

Hairston, Elaine (1996). "A Picaresque Journey, Corporate Change, Technological Todal Waves and Webby Worldviews". Change: A Journal of Higher Education, 28 (2) March/ April: 32-37. 
Hittz, S. R., y Johnson, K. (1990). "User Satisfaction with Computer-Mediated Communication Systems" en CALDWELL, Barrett y TAHA, Lilas (1993).

Internet Software Consortium (1999) en Matthew Ciolek: Global Networking:a Timeline 1990-1999_http://www.ciolek.com/PAPERS/GLOBAL/1900late.html\#1999 (Junio 5, 2000).

Internet Software Consortium (2000) en Matthew Ciolek: Global Networking:a Timeline 2000-2009. http://www.ciolek.com/PAPERS/GLOBAL2000t en.htmil\#2000 (Junio 5. 2000)

Lowry, T. (1992). (Entrevista con el autor) 15, 1992) en CALDWELL, Barrett y TAHA, Lilas (1993).

Maryniak, Nelson, J. A., y Caldwell, B. S. (1992). Experience, Utility and Situational Appropriateness: How Does Organizational Context Influence Usability of Electronic Communications Media? en CALDWELL, Barrett y TAHA, Lilas (1993).

Matthew Ciolek, T. (1998). "The Scholarly Uses of the Intemet: 1998 Online Survey". http://www.ciolek.com/PAPERS /InternetSurvey-98.html (Marzo 1999).

Mokhtarian, P.L. (1991). Telecommuting and Travel: State of the Practice, State of the Art en CALDWELL, Barrett y TAHA, Lilas (1993).

Papa, M.J. y Papa, W. H. (1990). "Perceptual and Communicative Indices of Employee Performance with New Technology" en CALDWELL, Barrett y TAHA, Lilas (1993).

Oison, M. H. (Ed,). (1989). Technological Support for Work Group Collaboration en CALDWELL, Barrett y TAHA, Lilas (1993).

Ouellette Kobasa, S. C., \& Puccetti, M. C. (1983). "Personality and Social Resources in Stress Resistance" en: CALDWELL, Barrett y TAHA, Lilas (1993).
Rice, R. E. (1984). "Mediated Group Communication" en CALDWELL, Barrett y TAHA, Lilas (1993).

Rice, R. E. y Shook, D.E. (1988). "Access to, Usage of, and Outcomes from an Electronic Messaging System" en CALDWELL, Barrett y TAHA, Lilas (1993).

Rice, R. E., y William S., F. (1984), "Theories Old and New: The Study of New Media" en CALDWELL, Barrett y TAHA, Lilas (1993).

Rice, R. E., Grant, A. E., Schmit Z, J. y Torobin, J. (1990). "Individual and Network Influences on the Adoption and Perceived Outcomes of Electronic Messaging" en CALDWELL, Barrett y TAHA, Lilas (1993).

Salom, Rosa (1999). The Venezuelan Universities and the Cyberspace. Paper presented at ED-MEDIA 99, Seattle, Washington, USA.

Sauter, S. L., Murphy, L. R., \& Hurrell, J. J. Jr. (1990). "Prevention of Work-Related Psychological Disorders: A National Strategy Proposed by the National Institute for Occupational Satety and Health (NIOSH) "en CALDWELL, Barrett y TAHA, Lilas (1993).

Schein, E.H. (1990). "Organizational Culture" en CALDWELL, Barrett y TAHA, Lilas (1993).

Short, J., Williams, E., \& Christie, B. (1976). The Social Psychology of Telecommunications en CALDWELL. Barrett y TAHA, Lilas (1993).

Silvio, J. (1998), "La Virtualización de las Universidades". Ponencia presentada en Visionarios 98, Caracas, Venezuela

Spears, R., Lea, M., \& Lee, S. (1990). "De-individuation and Group Polarization in Computer-mediated Communication" en CALDWELL, Barrett y TAHA, Lilas (1993).

Sproull, L., \& Kiesler, S. (1991). Connections: New Ways of Working in the Net- 
worked Organization en CALDWELL, Barrett y TAHA, Lilas (1993).

Sundstrom, E., Demeuse, K. P., \& Futrell, D. (1990). "Work Teams: Applications and Effectiveness" en: CALDWELL, Barrett y TAHA, Lilas (1993).

Taylor, J. C. (1987). Job Design and Quality of Working Life en CALDWELL, Barrett y TAHA, Lilas (1993).

Trevino, L. K., Lengel, R. H., \& Daft, R. L. (1987). "Media Symbolism, Media Richness, and Media Choice in Organizations: A Symbolic Interactionist Perspective" en CALDWELL, Barrett y TAHA, Lilas (1993).
Urribarri de Minardi, Raisa (1999). Uso de la Red Académica de la Universidad de los Andes. Una Indagación desde la perspectiva Comunicativa. Tesis de Grado para optar al título de Magister en Ciencias de la Comunicación y la Información. Facultad de Humanidades, Universidad del Zulia, Maracaibo.

Villalobos, Orlando (1998). Política y Gerencia de la Comunicación Social en la Universidad del Zulia. Tesis de Grado para optar al título de Magister en Ciencias de la Comunicación y la Información. Facultad de Humanidades, Universidad del Zulia, Maracaibo. 